\title{
FORMAÇÃO DE RECURSOS HUMANOS E PROMOÇÃO DA SAÚDE: UMA ANÁLISE DAS DIRETRIZES CURRICULARES NACIONAIS PARA A GRADUAÇÃO EM MEDICINA ${ }^{1}$
}

Leandro Januário de Lima, Graduando em Ciências Médicas na Universidade Federal de Campina Grande (UFCG), Bolsista do Conselho Nacional de Desenvolvimento Científico e Tecnológico (CNPq), leandrojanuario100@gmail.com

Roberto Ramon Queiroz de Assis, Graduando em História na Universidade Federal de Campina Grande (UFCG), roberto.ramon9@gmail.com

José Ferreira Lima Júnior, Doutor em Biotecnologia pela Universidade Federal da Paraíba (UFPB), Professor da Universidade Federal de Campina Grande (UFCG), ferreirajunior@cfp.ufcg.edu.br

\begin{abstract}
RESUMO
Buscou-se neste trabalho traçar um perfil da formação dos recursos humanos em saúde, notadamente os médicos, no que tange ao preparo para atuar na promoção da saúde. O problema foi investigado a partir de uma análise documental das diretrizes curriculares nacionais para a graduação em Medicina por este documento conter as primícias da organização do curso em nível nacional. Observou-se durante a pesquisa que embora as diretrizes já permeiem o assunto ainda é necessário avançar na inclusão deste conteúdo nos currículos além de integrá-lo a todos os níveis de atenção, pois, atualmente a promoção da saúde esta relacionada apenas à atenção primária à saúde. Concluiu-se que a pertinência do tema precisa ser revista e, sobretudo, ampliada na formação médica, para capacitar os futuros profissionais a atuarem de forma ativa nos determinantes sociais do processo saúde-doença superando os gargalos atuais.
\end{abstract}

PALAVRAS CHAVE: Promoção da Saúde; Recursos Humanos em Saúde; Educação de Graduação em Medicina.

\section{INTRODUÇÃO}

O conceito de saúde predominante determina-a como o produto de condicionantes diversos como o bem-estar físico, social e econômico, indo além de uma mera ausência de doenças e comorbidades. Neste sentido, a qualidade de vida da população entra em cena como determinante do processo saúde-doença corroborando então para o surgimento do conceito de promoção da saúde, definida pela Organização Mundial de Saúde (OMS) como aquela que

${ }^{1} \mathrm{O}$ presente trabalho não contou com apoio/financiamento de nenhuma natureza para sua realização.

Revista de Pesquisa Interdisciplinar, Cajazeiras, n. 2, suplementar, p. 525 - 537, set. de 2017. 
"consiste en proporcionar a los pueblos los medios necesarios para mejorar su salud y ejercer un mayor control sobre la misma" (OMS, 1996, p. 367).

As atividades de promoção em saúde se consolidaram no fim do século XX a partir de conferências que questionavam o processo de medicalização dentro do setor saúde, propondo que além dos serviços de saúde já estabelecidos se pudesse influenciar em seus determinantes. Logo, difundiu-se o conceito de promoção da saúde como um olhar para os determinantes globais do processo saúde-doença, enquanto a prevenção estaria voltada a fatores particulares (SÍCOLI; NASCIMENTO, 2003).

Para o Ministério da Saúde, a promoção da Saúde é "uma das estratégias de produção de saúde que, articulada às demais estratégias e políticas do Sistema Único de Saúde, contribui para a construção de ações transversais que possibilitem atender às necessidades sociais em saúde" (BRASIL, 2012). Esta concepção é fruto do desenvolvimento do conceito de promoção à saúde, que está fundamentada no axioma de que a saúde é produto dos diversos fatores sociais, mas que diferentemente da prevenção busca expandir o potencial positivo da saúde para além da mera ausência de enfermidades, buscando a efetivação de um estado de bem estar (SUCUPIRA; MENDES, 2003).

Neste sentido, tem se intensificado as ações e iniciativas concebidas no intuito de promover qualidade de vida, sobretudo no âmbito de políticas públicas (BUSS, 2000). Estas atividades de promoção da saúde foram concretizadas na política nacional de promoção da saúde que visava no âmbito institucional intervir para reduzir vulnerabilidades e riscos à saúde. Enquanto política de governo, os objetivos planejados envolveram as três esferas de governo e demandaram recursos financeiros e humanos, estes últimos determinantes no sucesso ou fracasso das atividades propostas (BRASIL, 2010; BRASIL, 2014).

Diante deste cenário a formação de recursos humanos em saúde capazes de atuar tanto na prevenção de agravos quanto na promoção de qualidade de vida da população é fundamental, sobretudo em uma nação plural e de enorme extensão territorial e que conta com um sistema universal de saúde a presença destes profissionais é importante para a consolidação os princípios de equidade e universalidade do atendimento (PAIM et al., 2011; NORONHA; LIMA; MACHADO, 2012). Assim, levando em consideração o longo tempo de formação na academia dos discentes dos cursos da área de Ciências da Saúde, é necessária a implantação de atividades dentro do currículo que conduzam a compreensão do desafio de 
consolidar um atendimento com integralidade, ferramenta ímpar para subsidiar a promoção da saúde

Partindo do cenário atual, Silva et al. (2012) destacam que os estudantes atuais possuem dificuldades de desenvolver um olhar integral do paciente por suas formações supervalorizarem um modelo de atuação tecnicista além dos discentes possuírem baixa adesão a conteúdos teórico-políticos. Por conseguinte, as diretrizes curriculares nacionais (DCNs) para os cursos de graduação - instituídas pela Lei de Diretrizes e Bases da Educação em 1996 - foram responsáveis por introduzir algumas mudanças nos currículos que estão auxiliando uma mudança na visão dos novos profissionais quanto a integralidade do serviço, princípio constitucional norteador das políticas de saúde (CECCIM; FEUERWERKER, 2004).

Logo, sabendo da importância da contribuição do médico para o desenvolvimento de ações de melhoria da qualidade de vida no contexto da Política Nacional de Promoção de Saúde no Sistema Único de Saúde é importante interrogar: como as diretrizes nacionais curriculares para o curso de Medicina abordam a questão da promoção da saúde? Neste sentido, determinar o quanto estes documentos norteadores da educação médica desenvolvem dentro de sua abrangência o fomento a construção de competências e habilidades no campo da promoção da saúde auxilia a direcionar o processo socioeducativo destes profissionais para uma formação com visão holística e integrativa do paciente enquanto sujeito. Assim, partindo da análise das diretrizes curriculares nacionais para o curso de Medicina dos anos de 2000 e 2014, este estudo objetiva traçar um perfil da formação dos recursos humanos em saúde, notadamente os médicos, no que tange ao preparo para atuar na promoção da saúde.

\section{MÉTODO}

Este estudo trata-se de uma pesquisa descritiva quanto aos seus objetivos, de cunho qualitativo, constituindo-se como pesquisa documental no seu princípio metodológico. Embora a pesquisa documental seja considerada como sinônimo da pesquisa bibliográfica por possuírem pontos em comum, como o uso de documentos em suporte físico e/ou eletrônico, a pesquisa documental possui como fonte de busca de informações os documentos que ainda não receberam tratamento analítico enquanto a pesquisa bibliográfica centra-se no contato com fontes científicas que lhe isentam do contato direto com o fenômeno empírico (SÁSILVA; ALMEIDA; GUINDANI, 2009; GERHARDT; FREITAS, 2009; MINAYIO, 2012). 
Prodanov e Freitas (2013, p. 56) conceituam como documento "qualquer registro que possa ser usado como fonte de informação". Ressaltam ainda que tais entidades devem ser submetidas a uma avaliação crítica do pesquisador que debruçar-se-á sobre seus aspectos internos e externos. Serão avaliadas nesta pesquisa as diretrizes curriculares nacionais para a graduação em Medicina. Comporão este rol as diretrizes publicadas nos anos de 2000 e 2014 pelo Conselho Nacional de Educação, tendo em vista que ambas foram publicadas após a promulgação da atual Lei de Diretrizes e Bases da Educação, ocorrida em 1996.

A análise das diretrizes curriculares nacionais configura esta pesquisa como documental por tratarem-se de documentos oficiais do Ministério da Educação bem como serem documentos jurídicos tipificados como resoluções. Elas configuram o que Gil (2008) elenca como documentos de segunda mão, pois, já sofreram algum grau de tratamento analítico sem, contudo, esgotar-se seu potencial documental. Seguiram-se nesta pesquisa as etapas da pesquisa documental definidas por Gil (2007), a saber: (i) determinação dos objetivos; (ii) elaboração do plano de trabalho; (iii) identificação das fontes; (iv) localização das fontes e obtenção do material; (v) tratamento dos dados; (vi) construção das fichas e redação do trabalho, e (vii) construção lógica e redação do trabalho.

Os documentos foram obtidos de forma eletrônica no endereço eletrônico do Ministério da Educação em maio de 2017 e partir de então se procedeu a sua análise com posterior redação do trabalho nos moldes aqui expostos.

\section{RESULTADOS}

\section{Diretrizes Curriculares Nacionais: resolução $\mathrm{CNE}^{\circ}{ }^{\circ}$ 4, de 7 de novembro de 2001}

As primeiras diretrizes instituídas para o curso definiram como perfil para o egresso uma formação generalista, humanista, crítica e reflexiva. A partir destas pilastras configurouse que o médico ao egressar da graduação deveria estar apto a exercer suas habilidades nos diversos níveis de atenção. Foi estipulado então que o profissional formado a partir das competências e habilidades desenvolve não somente ações de recuperação e reabilitação, mas também de promoção da saúde atuando numa visão integral do ser humano.

O predomínio, ou pelo menos a presença, desta visão holística do paciente enquanto sujeito é um dos marcos desta diretriz que expressa o anseio da Constituição Nacional no que tange ao fortalecimento da promoção da saúde bem como simultaneamente quebra os paradigmas impostos pelo modelo flexeneriano de educação médica que influencia até hoje as 
escolas médicas no que tange a fragmentação do paciente e a hiperespecialização. A presença destes pontos na diretriz se refletiu na criação de disciplinas com uma visão mais humanística que proporcionam a oportunidade de ver o paciente de maneira integral e, desta forma, capacitando os discentes, por meio das competências profissionais propostas, para a promoção da saúde e do bem-estar (SANTOS et al., 2015).

Foram elencadas no artigo $4^{\circ}$ da normativa seis áreas gerais de agregação de competências: (i) atenção à saúde; (ii) tomada de decisão; (iii) comunicação; (iv) liderança; (v) administração e gerenciamento, e; (vi) educação permanente. Dentro do primeiro conjunto foi citada tanto a capacidade de desenvolver ações de promoção da saúde em nível individual e coletivo como é direcionado que a prática do médico deva ser exercida com pensamento crítico e análise dos problemas sociais para serem elencadas soluções para os mesmos.

Das vinte e duas competências listadas no quinto artigo, seis estão de forma direta ou indireta ligadas as promoções da saúde dentro dos currículos e treinamento para a prática profissional. A primeira delas é clara ao estabelecer que o egresso deve "promover estilos de vida saudáveis, conciliando as necessidades tanto dos seus clientes/pacientes quanto às de sua comunidade, atuando como agente de transformação social.” (BRASIL, 2001, p. 38). Ainda é reforçada a tese que diante de um tratamento o profissional em formação adquira a capacidade de educar os parentes e cuidadores do paciente a executarem ações de promoção de saúde.

Ainda dentro das competências elencadas é proposto que a formação exponha a saúde enquanto um direito humano, imbuindo no profissional a capacidade de garantir a integralidade da assistência. Desta forma, o formando poderá "atuar na proteção e na promoção da saúde e na prevenção de doenças, bem como no tratamento e reabilitação dos problemas de saúde e acompanhamento do processo de morte" (BRASIL, 2001, p. 38).

Dentro dos conteúdos elencados como essenciais no artigo $6^{\circ}$ a promoção da saúde esta implícita dentro das noções de determinação social do processo saúde-doença além da integração do ensino-serviço com a ocorrência e incidência das morbidades proporcionando a integralidade do cuidar. Assim, promoção da saúde e processos fisiológicos do ser humano devem ser articuladas no currículo proporcionando uma formação integrada.

\section{Novas Diretrizes: resolução no 3 do CNE, de 20 de junho de 2014}

Estruturalmente mais extensa e com um número de seções maior, a resolução que instituiu as novas diretrizes curriculares em 2014 inicia no seu terceiro artigo um 
tangenciamento ao tema da promoção da saúde com a transversalidade entre a integralidade do atendimento com a determinação social do processo saúde doença.

Desta vez a formação médica que na diretriz anterior estava dividida em seis pequenos blocos de atuação agora se estrutura em três áreas que guiam a discussão da normativa sobre o desenvolvimento de competências e habilidades nos discentes, sendo elas: (i) atenção à saúde; (ii) gestão em saúde, e; (iii) educação em saúde. Dentre as três, aquela que mais tem um envolvimento direto com a capacitação para a promoção da saúde é a de atenção à saúde.

$\mathrm{O}$ quinto artigo trata dos componentes que devem ser considerados pelos formandos nas suas práticas profissionais, destacando-se a diversidade biológica, étnica, de gênero, socioeconômica, política, ambiental. Negligenciar uma destas põe desequilíbrio no objetivo de concretizar um atendimento mais humanizado e integral, que por sua vez não proporciona bem-estar ao paciente e refuta a promoção da saúde.

Destaca-se na visão da normativa que é preciso concretizar "promoção da saúde, como estratégia de produção de saúde, articulada às demais políticas e tecnologias desenvolvidas no sistema de saúde brasileiro, contribuindo para construção de ações que possibilitem responder às necessidades sociais em saúde" (BRASIL, 2014, p. 9).

Dentro das ações da seção de gestão em saúde, a valorização da vida desde a atenção até serviços de urgência e emergência é vista como indutora de promoção da saúde visando a melhoria da qualidade de vida. Além disso, a participação social e promoção da equidade nos serviços de saúde são elencadas como mecanismos de promoção de uma atenção contínua, integral e de qualidade. A seção de educação em saúde não contemplou de maneira direta a promoção da saúde, embora seja um dos componentes desta.

No capítulo II da resolução estão presentes competências pertinentes ao egresso, ESTANDO novamente distribuídas nas três áreas do capítulo anterior. Como consequência, a seção de competências de atenção à saúde encontra-se com um número maior de menções. Desta vez a área foi subdividida para elencar competências de atenção à saúde individual e coletiva.

As ações de promoção de saúde em âmbito individual incluem a prescrição de tratamentos com inclusão de práticas terapêuticas de cunho popular que tenham sido testadas e não causem dano, caso seja uma reivindicação do paciente ou cuidador. Além disso, o prognóstico dos pacientes deve levar em consideração seus contextos particulares, conforme consta no artigo 12. Já no âmbito coletivo, a diretriz instrui no seu artigo 14 que a graduação 
deve oferecer "estímulo à inserção de ações de promoção e educação em saúde em todos os níveis de atenção, com ênfase na atenção básica, voltadas às ações de cuidado com o corpo e a saúde" (BRASIL, 2014, p. 10). Dentro da seção de gestão em saúde o tema da promoção só é contemplado do ponto de vista da integralidade do entendimento no serviço no artigo 18.

Os conteúdos essenciais da nova resolução que permeiam a promoção da saúde em nada diferem daqueles expressos nas diretrizes de 2001. O artigo 23 novamente relata a determinação social do processo saúde doença e a relação entre processos fisiológicos e promoção da saúde.

\section{DISCUSSÕES}

A inclusão da temática da promoção da saúde nos currículos médicos no Brasil é fruto de um movimento iniciado na publicação da famosa história natural das doenças de Leavell e Clarck em 1976 que aqui teve como correspondente a reforma sanitária (GIOVANELLA, 2012; PAIM, 2008). Por isso, embora o Brasil forneça um serviço de saúde ainda muito centrado nas concepções flexenerianas, os currículos médicos já começaram a incorporar as temáticas de prevenção, promoção da saúde, humanização do cuidado e integralidade do atendimento, sobretudo com a instituição das diretrizes curriculares, culminando desta forma em uma educação mais voltada às necessidades sociais em saúde como proposto nas resoluções (AMORETTI, 2005).

Outro diferencial encontrado nas diretrizes foi a proposta de orientação de um currículo por competências e habilidades a serem desenvolvidas pelos futuros profissionais. Embora sejam propostos alguns conteúdos apontados como essenciais onde a promoção da saúde também esta presente é nas competências de ambas as diretrizes que a temática ganha destaque. A opção pelo currículo em competências se dá por possibilitar aos egressos maior eficácia no trato das questões pertinentes à realidade da saúde brasileira (FRANCO; CUBAS; FRANCO, 2014). É perceptível pelos trechos dos documentos que a humanização e a compreensão da integralidade ainda são desafios, e estudos apontam os reflexos tanto nos estudantes quanto egressos (RIOS; SIRINO, 2015; TAROCO; TSUJI; HIGA, 2017).

Ainda é incipiente a integralidade nas atividades de formação dos futuros médicos. Um dos desafios na sua prática é questão da interdisciplinaridade que se encontra deficiente nos cenários acadêmicos de formação e na prática profissional. Este é um dos pontos 
tangenciado nas diretrizes e que impacta diretamente nas ações desenvolvidas pelos profissionais no futuro no âmbito do sistema de saúde (SANTOS et al., 2015).

A promoção da saúde já foi incorporada pelos docentes como um componente necessário da matriz curricular das graduações em Medicina, indo ao encontro das diretrizes (GONTIJO et al., 2013). Ainda assim, prevalece um teor de resistência de alguns formadores em trabalhar com estas "novas" temáticas pelos mais diversos motivos, corroborando para os achados das diretrizes de 2014, onde embora decorrido um tempo considerável da instituição da normativa de 2001 houve poucos avanços, estes centrados principalmente na atenção primária à saúde (OLIVEIRA et al., 2008; VIEIRA; TAMOUSAUSKAS, 2013; NORMAN, 2013).

Como produto principal deste cenário tem-se a formação de profissionais, que por não terem contado com atividades que os direcionassem de forma suficiente ao contato com a promoção da saúde em meio acadêmico, com dificuldades em elencar dentro da sua prática profissional a humanização, integralidade e, por conseguinte, a promoção da saúde do paciente e seu bem-estar (HEIDEMANN; WOSNY; BOEHS, 2014). Uma provável mudança possa advir da obrigatoriedade da adesão às metodologias ativas de ensino-aprendizagem na graduação em Medicina, indicada pelas diretrizes de 2014. O contato com a problematização junto a inserção precoce no serviço defendidos pela normativa podem colaborar para um afloramento das competências de promoção da saúde por um desenvolvimento de uma cultura holística de atendimento (MAIA, 2014; FARIAS; MARTIN; CRISTO, 2015).

\section{CONSIDERAÇÕES FINAIS}

Constatou-se neste trabalho que desde as primeiras diretrizes existe a presença de ações e competências que visam formar profissionais com capacidade de atuar na promoção da saúde. Destacaram-se a presença de habilidades previstas em alguns dos setores das diretrizes curriculares nacionais além da presença da temática dentro dos conteúdos essenciais tanto em 2001 quanto em 2014.

É preciso ainda ampliar as noções propostas, pois, até o momento estão intimamente associadas às atividades na atenção primária à saúde e o desenvolvimento do bem-estar do paciente deve permear todos os níveis de atenção. Com a proposição de um currículo baseado em competências em ambas as resoluções é possível vencer os obstáculos que ainda se 
interpõem entre a formação de recursos humanos e o desenvolvimento de um atendimento humanizado com uma visão holística do paciente.

Assim, concluí-se que as diretrizes curriculares nacionais para o curso de graduação em Medicina já contemplam mecanismos que auxiliem no surgimento de profissionais atuantes na determinação social do processo saúde-doença muito além do binômio biológico, promovendo assim o bem-estar dos pacientes. Com a devida superação dos gargalos expostos conseguir-se-á atingir a meta presente nas próprias diretrizes, onde a academia fornecerá à sociedade um profissional capacitado a atuar na prevenção, terapêutica e promoção da saúde.

\title{
TRAINING OF HUMAN RESOURCES AND HEALTH PROMOTION: AN
}

\section{ANALYSIS OF NATIONAL CURRICULAR GUIDELINES FOR GRADUATION IN MEDICINE}

\begin{abstract}
This study aimed to outline a profile of the training of human resources in health, especially physicians, in relation to the preparation to act in the promotion of health. The problem was investigated from a documentary analysis of national curricular guidelines for medical graduation by this document containing the first fruits of the organization of the course at the national level. It was observed during the research that although the guidelines already permeate the subject, it is still necessary to make progress in the inclusion of this content in the curricula, besides integrating it at all levels of attention, since, currently, health promotion is related only to primary health care. It was concluded that the pertinence of the topic needs to be reviewed and, above all, expanded in medical training, to enable future professionals to actively participate in the social determinants of the health-disease process, overcoming the current bottlenecks.
\end{abstract}

KEYWORDS: Health Promotion; Human Resources in Health; Graduate Education in Medicine.

\section{FORMACIÓN DE RECURSOS HUMANOS Y PROMOCIÓN DE LA SALUD: UN ANÁLISIS DE LAS DIRECTRICES CURRICULARES NACIONALES PARA LA GRADUACIÓN EN MEDICINA}

\section{RESUMEN}

Se buscó en este trabajo trazar un perfil de la formación de los recursos humanos en salud, especialmente los médicos, en lo que se refiere a la preparación para actuar en la promoción de la salud. El problema fue investigado a partir de un análisis documental de las directrices curriculares nacionales para la graduación en Medicina por este documento contener las primicias de la organización del curso a nivel nacional. Se observó durante la investigación 
que aunque las directrices ya permean el asunto aún es necesario avanzar en la inclusión de este contenido en los currículos además de integrarlo a todos los niveles de atención, pues, actualmente la promoción de la salud esta relacionada apenas a la atención primaria a la Salud. Se concluyó que la importancia del tema necesita ser revisada y, sobre todo, ampliada en la formación médica, para capacitar a los futuros profesionales a actuar de forma activa en los determinantes sociales del proceso salud-enfermedad superando los cuellos de botella actuales.

PALABRAS CLAVE: Promoción de la Salud; Recursos Humanos en Salud; Educación de Graduación en Medicina.

\section{REFERÊNCIAS BIBLIOGRÁFICAS}

AMORETTI, R. A Educação Médica Diante das Necessidades Sociais em Saúde. Revista Brasileira de Educação Médica. Rio de Janeiro, v. 29, n. 2, p. 136-146, maio/ago. 2005.

BRASIL. Conselho Nacional de Secretários de Saúde. Avaliação em promoção da saúde: uma antologia comentada da parceria entre o Brasil e a cátedra de abordagens comunitárias e iniquidades em saúde (CACIS), da Universidade de Montreal de 2002 a 2012. Brasília: CONASS, 2014.

BRASIL. Ministério da Educação. Conselho Nacional de Educação. Resolução nº 4, de 07 de novembro de 2001. Institui Diretrizes Curriculares Nacionais do Curso de Graduação em Medicina. Diário Oficial da União. Brasília, 09 nov. 2001. p. 38-39.

BRASIL. Ministério da Educação. Conselho Nacional de Educação. Resolução n ${ }^{0}$ 3, de 20 de junho de 2014. Institui Diretrizes Curriculares Nacionais do Curso de Graduação em Medicina e dá outras providências. Diário Oficial da União. Brasília, 23 jun. 2014. n. 117, Seção 1, p. 8-11.

BRASIL. Ministério da Saúde. Glossário temático: promoção da saúde. Brasília: Ministério da Saúde, 2012. 48 p.

BRASIL. Ministério da Saúde. Política Nacional de Promoção da Saúde. 3. ed. Brasília: Ministério da Saúde, 2010.

BUSS, P. M. Promoção da saúde e qualidade de vida. Ciência \& Saúde Coletiva, v. 5, n. 1, p. 163-177, jan./mar. 2000. 
CECCIM, R. B.; FEUERWERKER, L. C. M. Mudança na graduação das profissões de saúde sob o eixo da integralidade. Cad. Saúde Pública, Rio de Janeiro, v. 20, n. 5, p. 1400-1410, set-out, 2004.

FARIAS, P. A. M.; MARTIN, A. L. A. R.; CRISTO, C. S. Aprendizagem Ativa na Educação em Saúde: Percurso Histórico e Aplicações. Revista Brasileira de Educação Médica, Rio de Janeiro, v. 39, n. 1, p. 143-150, Mar. 2015.

FRANCO, C. A. G. S.; CUBAS, M. R.; FRANCO, R. S. Currículo de medicina e as competências propostas pelas diretrizes curriculares. Revista Brasileira de Educação Médica, Rio de Janeiro, v. 38, n. 2, p. 221-230, Jun. 2014.

GERHARDT, T. G.; SILVEIRA, D. T. (Orgs.). Métodos de pesquisa. Porto Alegre: Editora da UFRGS, 2009.

GIL, A. C. Como elaborar projetos de pesquisa. 4. ed. São Paulo, Atlas, 2007.

GIL, A. C. Métodos e técnicas de pesquisa social. 6. ed. São Paulo: Atlas, 2008.

GIOVANELlA, L. (Ed.). Políticas e Sistemas de Saúde no Brasil. 2. ed. Rio de Janeiro: FIOCRUZ, 2012. 1100 p.

GONTIJO, E. D. et al. Matriz de competências essenciais para a formação e avaliação de desempenho de estudantes de medicina. Revista Brasileira de Educação Médica, Rio de Janeiro, v. 37, n. 4, p. 526-539, Dez. 2013.

HEIDEMANN, I. T. S. B.; WOSNY, A. M.; BOEHS, A. E. Promoção da Saúde na Atenção Básica: estudo baseado no método de Paulo Freire. Ciência e Saúde Coletiva, Rio de Janeiro, v. 19, n. 8, p. 3553-3559, Ago. 2014.

MAIA, J. A. Metodologias problematizadoras em currículos de graduação médica. Revista Brasileira de Educação Médica, Rio de Janeiro, v. 38, n. 4, p. 566-574, Dez. 2014.

MINAYO, M. C. S. Análise qualitativa: teoria, passos e fidedignidade. Ciência e saúde coletiva, Rio de Janeiro, v. 17, n. 3, p. 621-626, Mar. 2012.

NORMAN, A. H. Promoção da saúde: um desafio para a atenção primária. Revista Brasileira de Medicina de Família e Comunidade, Rio de Janeiro, v. 28, n. 8, p. 153-154, jul./set. 2013. 
NORONHA, J. C.; LIMA, L. D. MACAHDO, C. V. O Sistema Único de Saúde - SUS. In: GIOVANELlA, L. (Org.). Políticas e Sistemas de Saúde no Brasil. 2. ed. Rio de Janeiro: FIOCRUZ, 2012.

OMS. Organización Mundial de la Salud. Carta de Ottawa para la promoción de la salud. In: ORGANIZACIÓN PANAMERICANA DE LA SALUD. Promoción de la salud: uma antologia. Washington: OPAS, 1996.

PAIM, J. et al. The Brazilian health system: history, advances, and challenges. The Lancet, [s.1.], v. 377, n. 9779, p.1778-1797, maio 2011. Elsevier BV. http://dx.doi.org/10.1016/s0140-6736(11)60054-8.

PAIM, J. S. Reforma Sanitária Brasileira: contribuição para a compreensão e a crítica. Salvador: EDUFBA; Rio de Janeiro: FIOCRUZ, 2008.

PRODANOV, C. C; FREITAS, E. C. Metodologia do Trabalho Científico: métodos e técnicas da pesquisa e do trabalho acadêmico. 2. ed. Novo Hamburgo: Freevale, 2013.

RIOS, I. C.; SIRINO, C. B. A Humanização no Ensino de Graduação em Medicina: o Olhar dos Estudantes. Revista Brasileira de Educação Médica, Rio de Janeiro, v. 39, n. 3, p. 401409, Set. 2015.

SANTOS, R. N. L. C. et al. Integralidade e Interdisciplinaridade na Formação de Estudantes de Medicina. Revista Brasileira de Educação Médica, Rio de Janeiro, v. 39, n. 3, p. 378387, Set. 2015.

SÁ-SILVA, J. R.; ALMEIDA, C. D.; GUINDANI, J. F. Pesquisa documental: pistas teóricas e metodológicas. Revista Brasileira de História e Ciências Sociais, v. 1, n. 1, p. 1-15, jul. 2009.

SÍCOLI, J. L., NASCIMENTO, P. R. Health promotion: concepts, principles and practice. Interface - Comunic., Saúde, Educ., v. 7, n. 12, p.101-122, fev. 2003.

SILVA, E. N. et al. Formação de recursos humanos: um desafio para a promoção da saúde. In: OLIVEIRA, F. C.; LIMA JÚNIOR, J. F.; MOREIRA, M. R. C. (Orgs.). Resgatando Saberes e Ressignificando Práticas. Interfaces no campo da Saúde Coletiva. Campina Grande: EDUFCG, 2012.

SUCUPIRA, A. C.; MENDES, R. Promoção da saúde: conceitos e definições. SANARE, v. 4, n. 1, p. 7-10, Jan./Mar., 2003. 
TAROCO, A. P. R. M.; TSUJI, H.; HIGA, E. F. R. Currículo Orientado por Competência para a Compreensão da Integralidade. Revista Brasileira de Educação Médica, Rio de Janeiro, v. 41, n. 1, p. 12-21, Jan. 2017.

VIEIRA, J. E.; TAMOUSAUSKAS, M. R. G. Avaliação das resistências de docentes a propostas de renovações em currículos de graduação em medicina. Revista Brasileira de Educação Médica, Rio de Janeiro, v. 37, n. 1, p. 32-38, Mar. 2013. 